DEFORMABLE GRATING LIGHT VALVES

FOR HIGH RESOLUTION DISPLAYS

Raj B. Apte, Francisco S. A. Sandejas,

William C. Banyai, and David M. Bloom

\author{
Ginzton Laboratory \\ Stanford University \\ Stanford, CA 94305-4085
}

\section{Up: Reflection}

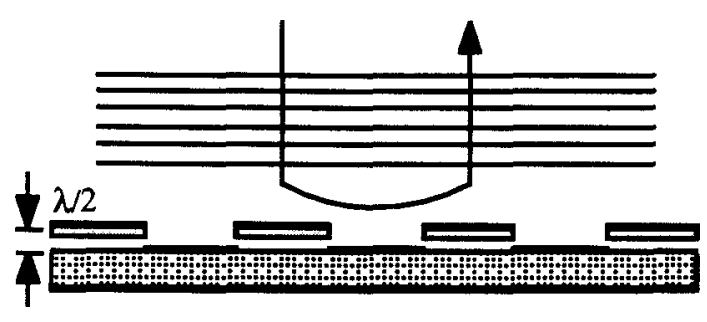

Silicon nitride beam

The Grating Light Valve (GLV) is a micromechanical phase grating that can be used for black\&white or color display applications. Operation is based on electrically controlling the mechanical positions of the grating elements to modulate the diffraction efficiency. Proper choice of the grating dimensions allows the structure to operate as a digital optical device.

Since the grating is inherently dispersive, the GLV can be used for color displays. In addition, the devices are bistable and may be able to operate with a passive matrix of contacts and still achieve the performance of an active matrix light valve. Eight bits of gray scale are possible using time division multiplexing and the fast (20 ns) switching speed of the GLV. The contrast ratio of the device is very sensitive to processing errors; to date, a ratio of $20: 1$ has been measured. With better processing, a color contrast of 200:1 should be achieved for a monochrome pixel.

Stiction of the micromachined grating elements to the substrate during the final wet processing step and during operation remains a problem. However, our current approach uses rough (150 $\AA$-RMS) polysilicon films to reduce the area of contact. When doped, the polysilicon can be patterned to function as a bottom electrode for twodimensional array applications. Although this structure suffers from some of the difficulties of a non-planar process, it was used to demonstrate two-dimensional arrays of devices with switching voltages as low as $11 \mathrm{~V}$.

\section{Introduction:}

The basic principle of operation for the GLV is illustrated in figure 1. The structure is a reflection phase grating consisting of aluminum overcoated silicon nitride microbridges. With no voltage applied, the total path length difference between light reflected from the microbridges and the substrate equals the wavelength of incoming light. These reflections add in phase and the grating reflects light as a flat mirror. When a voltage is applied between the microbridges and the substrate, the electrostatic force pulls the microbridges down.
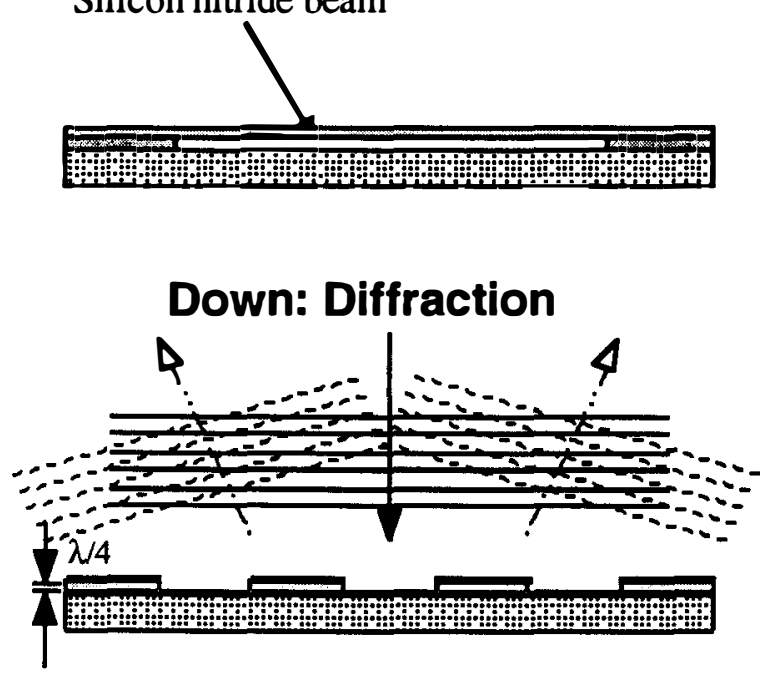

Silicon dioxide spacers

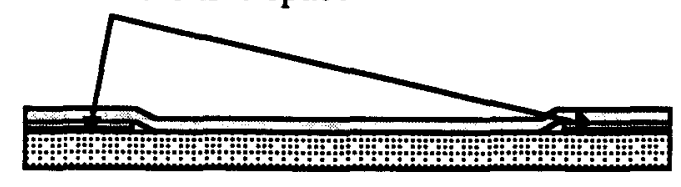

Figure 1: Operational principle of GLV.

Now the total path length difference between light reflected from the microbridges and the substrate is one-half wavelength, and the reflections interfere destructively causing light to be diffracted. For example, when a pixel consisting of 10 one-micron line-pairs in the down state is illuminated with normally incident white light, the \pm first-order diffraction beams have an angular bandwidth of approximately $4^{\circ}$ about $\pm 14^{\circ}$. Also, $42 \%$ of the light is diffracted into each of the first-order beams, so that an overall efficiency of $84 \%$ is possible when both beams are collected. Light in higherorder modes is diffracted at large angles and is not collected by the optical system.

\section{Optical Design:}

An example of a basic optical system for realizing a display is shown in figure 2 . It contains 


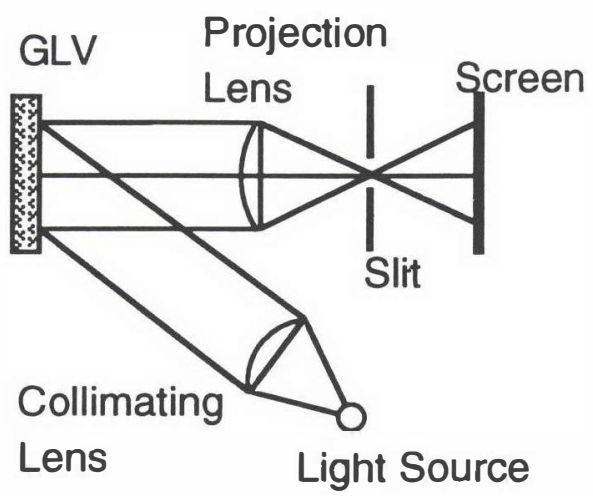

Figure 2: GLV Optical System.

a simple imaging system with unity magnification: the object plane containing the GLV array and the image plane containing the viewing screen are both $2 \mathrm{~F}$ from the lens, where $\mathrm{F}$ is the focal length. The GLV array is illuminated by a collimated light source and there is a spatial filter in the Fourier plane, i.e. one focal length in front of the lens. The GLV array is made up of individual pixels. Figure 3 is a micrograph of a $25 \mu \mathrm{m}$ by $25 \mu \mathrm{m}$ pixel consisting of 12 one-micron linepairs (a linepair is a one-micron microbridge and a one-micron space). The critical parameters in the system design are the number of linepairs defining a pixel, the degree of source collimation, and the width of the slit. Note that a slit is used because light is only diffracted in the plane perpendicular to both the microbridges and the plane of the pixel. The best way to understand the operation of the GLV system is to work through the three cases for realizing monochrome, black\&white, and color imaging.

For monochrome operation, we consider a point source of spectrally pure light, in this case green light at $\lambda_{\mathrm{g}}=530 \mathrm{~nm}$, that is collimated by a lens. With the GLV in the down state, the incident light is diffracted into the +first-order according to diffraction grating equation

$$
\sin \theta_{i}-\sin \theta_{d}=\lambda / p,
$$

where $\theta_{\mathrm{i}}$ is the incident angle, $\theta_{\mathrm{d}}$ is the diffraction angle, $\lambda$ is the wavelength, and $p$ is the spacing between the microbridges. The diffracted light is collected by the imaging lens and brought to a focus in the focal plane of the lens, since the diffracted light from the grating remains collimated, before continuing to the image plane where it interferes to form an image ${ }^{1}$. The distance from optical axis in the focal (Fourier) plane where the light comes to a focus is given by the ray tracing equation

$$
\partial X \cong \partial \theta \cdot F,
$$

where $F$ is the focal length of the lens. If we choose the microbridge spacing $\mathrm{p}=2.25 \mu \mathrm{m}$, the incident angle of collimated light $\theta_{\mathrm{i}}=13.6^{\circ}$ with respect to the optical axis, and $\lambda_{\mathrm{g}}=530 \mathrm{~nm}$, then the angle of the diffracted light is zero with respect to

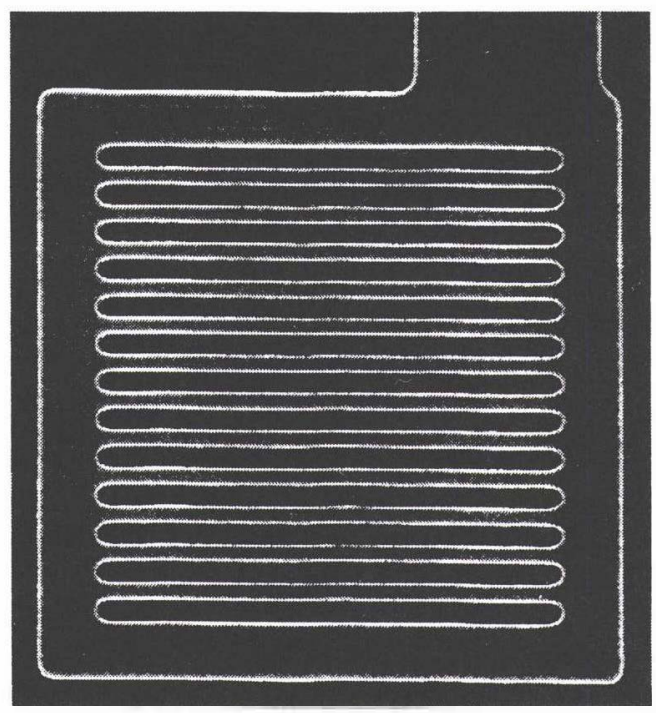

Figure 3: GLV $25 \mu \mathrm{m} \times 25 \mu \mathrm{m}$ pixel.

the optical axis. Therefore, all green light is brought to focus at the on-axis focal point of the imaging lens. We have assumed that all diffracted light is in the \pm first-order, however, in reality, a small amount of light is diffracted into the second and third orders. When the GLV is in the up state there is no diffraction and the light is specularly reflected at an angle $-13.6^{\circ}$ with respect to the optical axis, which will come to a focus $12 \mathrm{~mm}$ from the optical axis in the focal plane, according to equation (2). Now, the angular width of the diffracted light is given by 2

$$
\partial \theta \cong \lambda / D
$$

where $\mathrm{D}$ is the width of pixel. If we require the half-angular width of the diffracted light to be less than $13.6 \% / 2$ then $D \geq 2.23 \mathrm{~mm}$. Therefore, if we use one $1.125 \mathrm{~mm}$ linepairs $(D=2.25 \mathrm{~mm})$, then, using equation (3) to calculate the angular extent of the first-order beam and equation (2) to calculate its spatial extent in the focal plane of the lens, we find that a slit width of $11.8 \mathrm{~mm}$ will pass the diffracted light but block the reflected light. Notice that only a single linepair will function as an effective light valve. The contrast ratio, when measured as light through the slit when the microbridges are down to 
when they are up, will be unrealistically large due to our assumption of a spectrally-pure point source. In fact, the finite width of the source translates to angular error in the collimated incident light via equation (2). This error must be taken into account when calculating $\mathrm{D}$, resulting in an increase in $\mathrm{D}$, or number of linepairs. The finite extent of the source will have a considerable effect on the color operation described below.

For the second case of black\&white operation, we consider a whitelight point source. Two refinements are required in this case since the diffraction angle will be slightly different for the red and blue components and the GLV is perfectly reflecting in the up state only for the center green wavelength. The red components will be offset slightly to one side while the blue components are offset slightly to the other side of the green light in the focal plane. The offset can be calculated by differentiating equation (1) with respect to 1 , such that

$$
\partial \theta_{d}=-\partial \lambda / d \cos \theta_{d}
$$

Equation (4) can be used to calculate $\delta \theta$ and that value plugged into equation (2) to calculate $\delta \mathrm{X}$. For the system described above, the offset for blue light at $465 \mathrm{~nm}$ is $\delta \mathrm{X}_{\mathrm{b}}=1.4 \mathrm{~mm}$ while the offset for red light at $625 \mathrm{~nm}$ is $\delta \mathrm{X}_{\mathrm{r}}=2.1 \mathrm{~mm}$. The offset means that the number of linepairs must be increased to keep the spatial extent of the light in the focal plane to less than $11.8 \mathrm{~mm}$. Recalculating the pixel width by requiring the spatial extent of the red light to be less than $2 \times(11.8 / 2-2.1) \mathrm{mm}$ means that $\mathrm{D}=4.1 \mu \mathrm{m}$. This means that a minimum of two linepairs is required for black\&white operation of a GLV system. In fact, we only considered the center wavelengths of the red and blue light but should have considered the full bandwidth of the source used, usually from $400-700 \mathrm{~nm}$. The second consideration for black and white operation is the thickness of the microbridge and spacer. Our assumption was that the diffracted light turns on and off when the bridge move from down to up. In reality, this condition only holds at a single wavelength. In figure 4 , we see that when the microbridges are in the down position most of the light is diffracted into the first order with some of the light diffracted into the second and third orders. When the microbridges are up, then most of the light is in the specular mode and the first order is nulled. However, when the diffraction into the first-order is replotted in terms of wavelength about

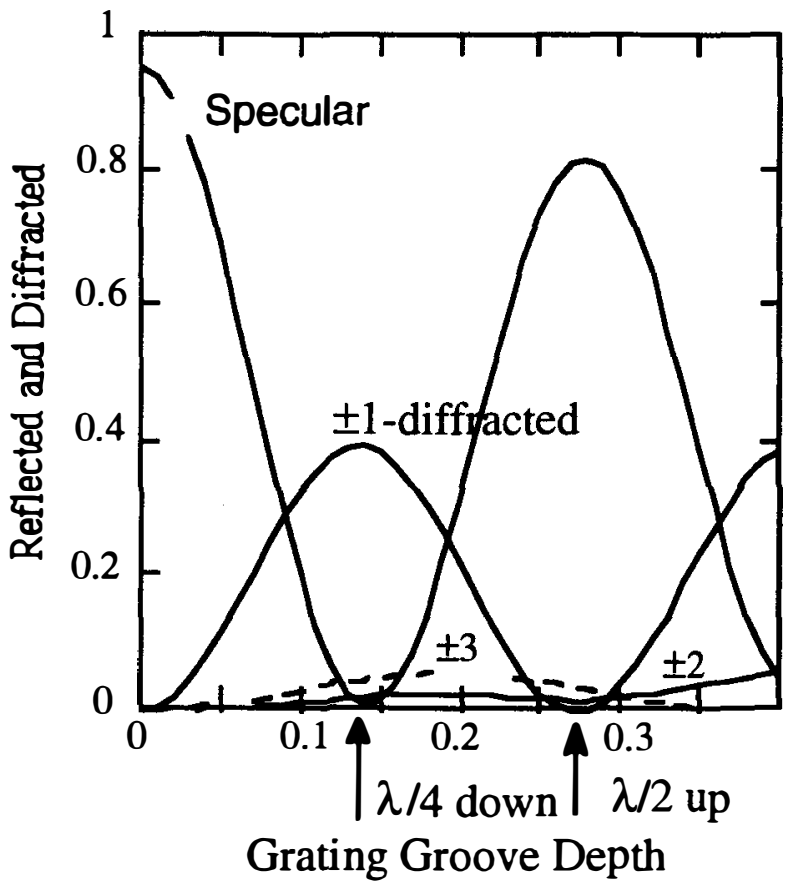

Figure 4: Diffraction efficiency ${ }^{3}$ for several orders of an aluminum reflection phase grating with 2.00 $\mu \mathrm{m}$ period, illuminated at normal incidence at $\mathbf{5 5 0}$ $\mathrm{nm}$. Note the finite reflectivity of aluminum limits the specular reflection with no groves to $92-94 \%$. [Gaither 1988; Veldkamp 1989]

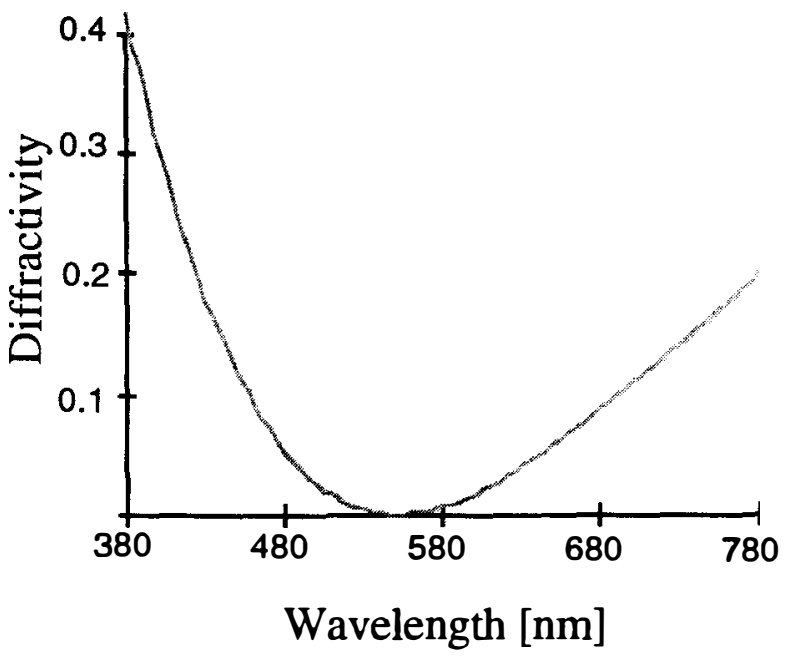

Figure 5: Diffractivity of the first order as a function of wavelength for a " up " pixel with a $550 \mathrm{~nm}$ design wavelength.

the $\lambda / 2$ groove depth, as in figure 5 , we see that some light is diffracted into the first order due to the finite bandwidth of the light source. The effect of 
this leakage is to limit the achievable contrast ratio to 82:1 for a whitelight source.

The third case to consider is when the GLV system is configured for color operation. In the previous examples it was shown that the angles at which the various colors are diffracted are determined solely by the grating period as indicated in equation (1). The movement of the microbridges only changes the amount of light diffracted but not the direction of diffraction. For the pixel described above with a period of $2.25 \mu \mathrm{m}$, the green light is diffracted normal to the GLV plane. We can now make similar pixels for the red and blue wavelengths by requiring $\theta_{\mathrm{d}}=0$ for $\theta_{\mathrm{i}}=13.6$. Using equation (1), we calculate $\mathrm{p}_{\mathrm{r}}=2.66 \mu \mathrm{m}$ and $\mathrm{p}_{\mathrm{b}}=1.97 \mu \mathrm{m}$. For the display shown in figure 1 , we now replace each green pixel with a red-green-blue triad, as shown in figure 6 . Color is then realized

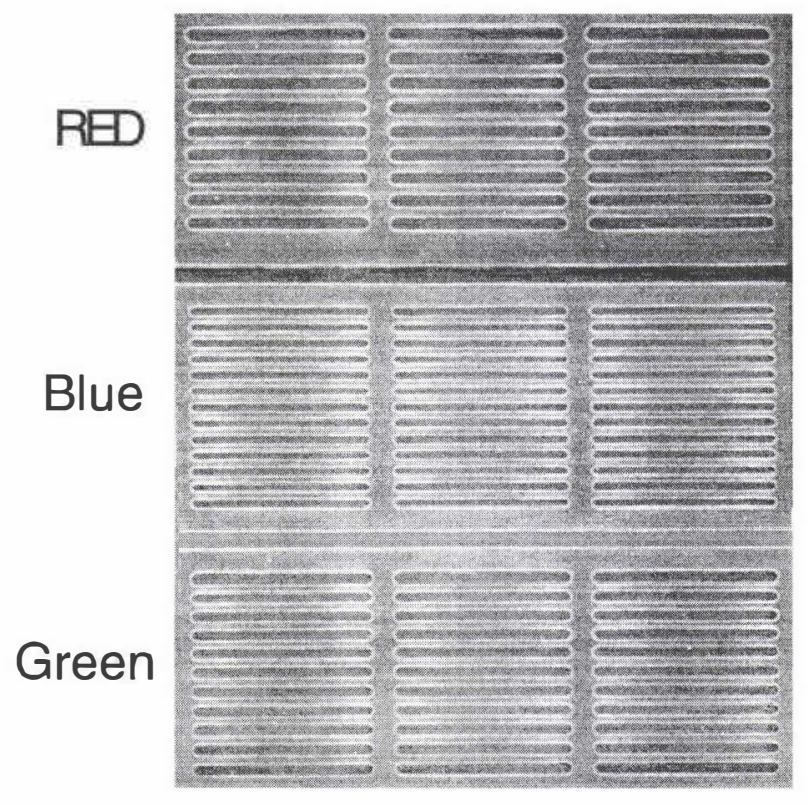

Figure 6: Red, blue and green pixels.

by reducing the slit width to only allow a limited bandwidth about each of the primary colors, as illustrated in figure 7. The slit width can be calculated by assuming a bandwidth, $80 \mathrm{~nm}$ for example, and using the above equations. The slit width for color operation of the above system is only $2 \mathrm{~mm}$. The number of linepairs also has to be recalculated to limit the angular extent of each color, as shown in figure 7 . Or, to put it another way, so that all of the light from the center wavelength passes through the slit. From the above equations, $\mathrm{D}=530 \mathrm{~nm} \times 50 \mathrm{~mm} / 2 \mathrm{~mm}=13 \mu \mathrm{m}$, or about 6 linepairs. This is the minimum number required. Using more linepairs translates to purer colors, which is why our designs tend to use 10-12 linepairs. There is also a tighter constraint on the spatial extent of the point source, since deviations from collimation allow unwanted color through the slit, reducing the color purity. An upper bound on the spatial extent of the source can be determined by assuming that the deviation in the focal plane due to collimation error in less than one-half the slit width. It turns out that when the focal lengths of the collimation lens and the imaging lens are equal the constraint translates directly to the source, so the source is limited to $1 \mathrm{~mm}$. The final consideration for color operation is the contrast ratio. With a triad of pixels the contrast ratio is degraded due to all three pixels sharing the same thickness of microbridge. For example, when the green pixel is off the red and blue pixels still diffract light, according to figure 5 . The red-green-blue contrast ratios change from the monochrome values of 20,341 , and 22 to triad values of 36,59 , and 6 . The red improves slightly due to the narrower slit used in the color GLV system.

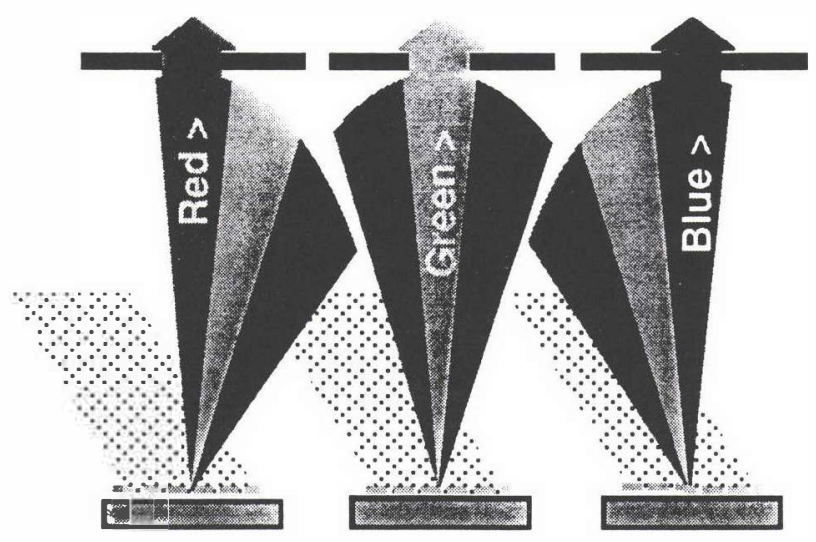

Figure 7: Color display approach: changing the grating period for a constant incident angle adjusts which color is diffracted normal to the surface.

\section{Beam Mechanics:}

The most striking feature of the mechanical operation of the GLV is the hysteresis of the deflection of the microbridges as a function of applied voltage. The reason for the hysteresis is that the electrostatic attraction between the top and bottom electrodes is a nonlinear function of the deflection while the restoring force caused by the stiffness and tension is linear, as illustrated in figure 8. The hysteresis curve for a single pixel is shown in figure 9. To generate this curve, the light diffracted into the +first-order was measured as a function of applied voltage. The switching voltage is approximately $20 \mathrm{~V}$ and is largely determined by the stress in the nitride microbridge as well as its length. In our earlier discussion, we calculated the number of linepairs which determined the width of the pixel, however, we did not discuss the reason 
for the length of the microbridge. It is determined by the desired usable surface area on the microbridge for diffraction when in the deflected state and the stiffness required for providing the restoring force. In fact, a longer structure requires more film stress to keep the restoring force for equal. Figure 10 illustrates the trend in switching voltages for two different length pixels. Low switching voltages ( $5 \mathrm{~V}$ range) are possible with 25 $\mu \mathrm{m}$ long microbridges, but at the expense of the restoring force used to overcome stiction.

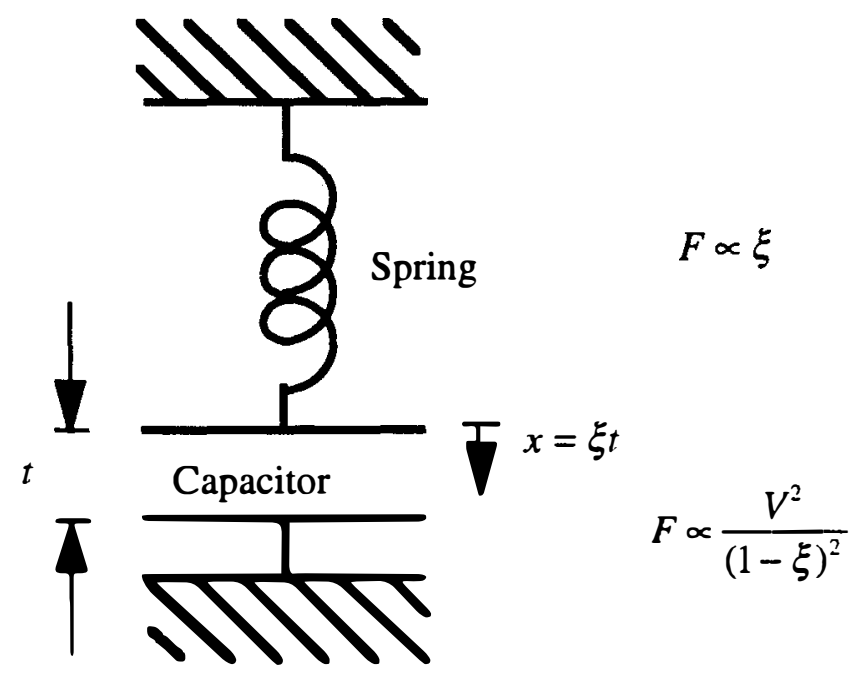

Figure 8: The basic model for the GLV beam mechanics. The spring represents the restoring force caused by the beam stiffness and tension. The capacitor represents the electrostatic attraction between the electrodes.

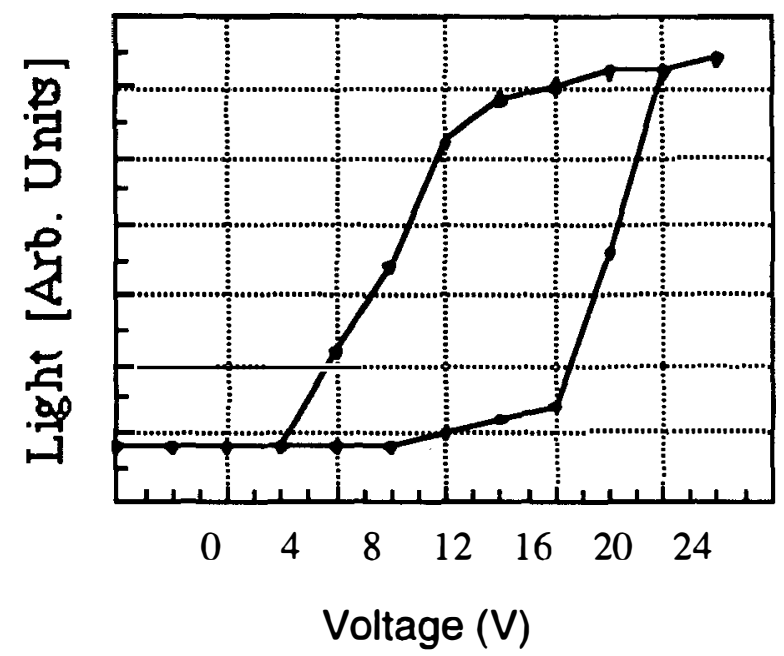

Figure 9: Hysteresis curve for pixel with $25 \mu \mathrm{m}$ long microbridges under $800 \mathrm{MPa}$ of tension.

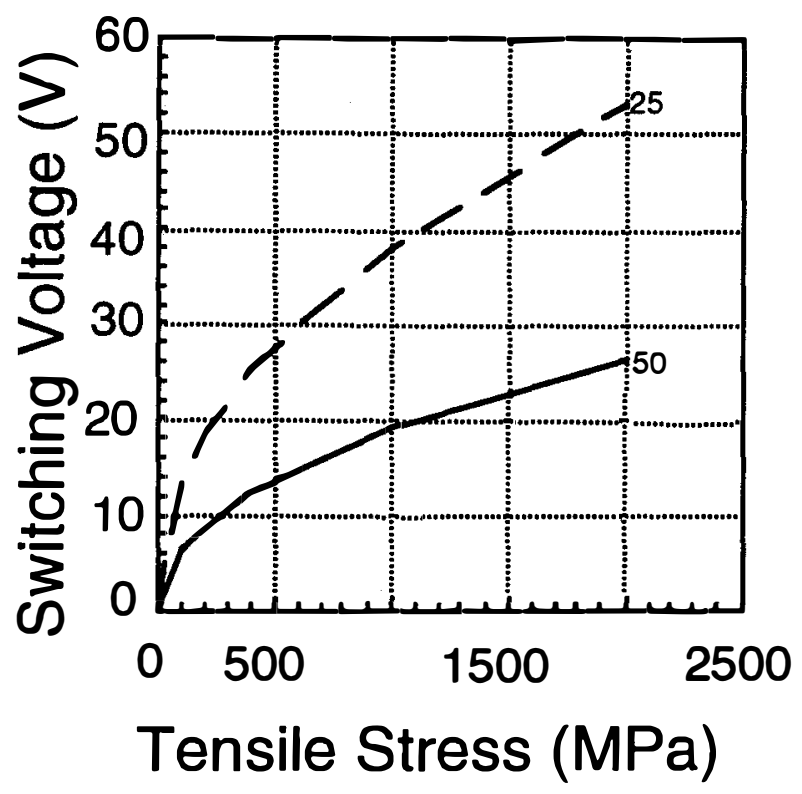

Figure 10: Switching voltage versus microbridge tension for two different lengths.

The other mechanical property of importance is the resonant frequency of the microbridge. When under tensile stress ${ }^{4}$,

$$
f_{\text {res }}^{\text {stress }}=\frac{1}{2 \pi L \sqrt{10 \frac{\sigma}{\rho}}},
$$

where $\rho$ is the density of the silicon nitride, $s$ is the stress, and $L$ is the length. This can be compared to the resonant frequency under low or no stress,

$$
f_{\text {res }}^{\text {nonstress }}=\frac{h}{2 \pi L^{2}} \sqrt{10 \frac{E}{\rho}}
$$

where E is Young's modulus. The ratio of these two resonant frequencies is

$$
\frac{f_{\text {res }}^{\text {stress }}}{f_{\text {res }}^{\text {nonstress }}}=\frac{L}{h} \sqrt{\frac{\sigma}{E}}
$$

For example with a stress of $\sigma=200 \mathrm{MPa}$, the resonant frequency increases by a factor of 3 over the nonstressed case to a value of $5.1 \mathrm{MHz}$. This fast response is important to the operation of the GLV since grayscale modulation can be implemented by pulsewidth modulation. However, from the preceding discussion, we note that there is a tradeoff between switching voltage and stress: the devices with the highest stress will be the fastest but will also suffer from large switching voltages. Our 
fastest measured switching time to date has been 20ns switching times for pixels with $20 \mu \mathrm{m}$ long microbridges under $800 \mathrm{MPa}$ of tension.

\section{Fabrication:}

The first step in fabrication of the device illustrated in figure 1 was to deposit on an I-Prime wafer $1325 \AA$ of silicon dioxide and $1325 \AA$ of silicon nitride. The nitride was patterned to form the frame and beams of the device. Then an isotropic, selective etch was used to undercut the oxide from beneath the beams. In order to free the beams, at least $0.75 \mu \mathrm{m}$ of undercut was needed. This was not enough, however, to completely remove the oxide from beneath the frame. In this way the frame remained supported by the oxide, and the beams were supported by the frame. Lastly, $400 \AA$ of aluminum was evaporated onto the top of the structure to form the top electrode and reflector. These devices suffer from the effects of stiction when released and operated, unless short $(15 \mu \mathrm{m})$ microbridges and high tension nitride were used, requiring drive voltages as high as $40 \mathrm{~V}$.

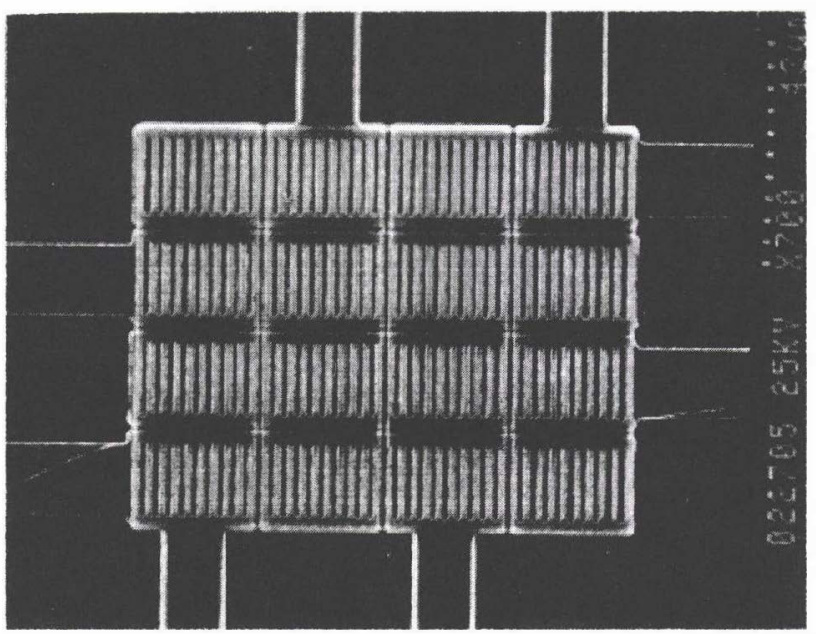

Figure 11: $4 \times 4$ array with polysilicon bottom electrodes.

Our more recent approach utilizes rough polysilicon films to overcome the sticking problem. This was successfully applied to the two dimensional array illustrated in figure 11 . Processing begins with a $5000 \AA$ oxide isolation layer being grown on a bare silicon substrate. On top of this layer a 3000-6000 ̊ undoped polysilicon layer was grown. The layer was probably amorphous initially, but it crystallizes during subsequent high temperature processing (the reflow step). The polysilicon was diffusion doped with phosphorous, cleaned, and patterned into the bottom interconnect layer. $1325 \AA$ of low temperature LPCVD oxide were deposited on top of the nonplanar bottom electrode traces. This oxide was doped with $8 \%$ phosphorous to reduce the reflow temperature. The oxide was steam reflowed at $1000{ }^{\circ} \mathrm{C}$ for 20 minutes. $1325 \AA$ of LPCVD nitride was deposited next, then patterned and dry etched. The wafer was then cleaned to remove all traces of photoresist and then released. The oxide spacer was removed from under the beams. In addition, oxide was removed from under the edges of the frames and the bottom electrode traces. The overhanging polysilicon and nitride provide the isolation. These devices switched at $11 \mathrm{~V}$ and did not exhibit stiction problems.

\section{Conclusion:}

We have given a general overview of the design of the GLV system with an emphasis on the critical optical constraints, namely, the number of linepairs required per pixel for optimum contrast ratio, the slit width and the source width. The salient feature of the GLV system is its inherent ability to be configured for color operation, at the expense of contrast ratio and loss of two -thirds of the optical power. Also, the basic microbridge mechanics were discussed to illustrate hysteresis and high-speed operation. Finally, twodimensional array fabrication was described.

\section{Acknowledgments:}

This research was supported by grants from ARPA/ARO DAAL03-92-G-0232 and ARPA/AFOSR F49620-93-I-0609.

\section{$\underline{\text { References: }}$}

1. For a more detailed explanation see Abbe's theory of image formation in Principles of Optics, Born, $M$ and E. Wolfe, Pergamon Press, New York, 1959.

2. See pages 61-63 in Introduction to Fouler Optics, Goodman, J. W., McGraw-Hill Book Company, San Francisco, 1968.

3. Gaither, S. A. "Two-Dimensional Diffraction from a Surface-Relief Grating," Computer Program, Vers. 26 May 1988, courtesy of W. VeldKamp, MIT Lincoln Laboratory; Veldkamp, W. B., G. J. Swanson, S. A. Gaither, C.-L. Chen, and T. R. Osborne, "Binary Optics: A Diffraction Analysis," MIT Lincoln Laboratory, Aug. 23, 1989. ODT-20.

4. Solgaard, O., "Integrated Semiconductor Light Modulators for Fiber-Optic and Display Applications", Ph.D. Thesis, Stanford U , 1992. 